\title{
Recurrent cornual ectopic pregnancies
}

\author{
Sitamani Sahoo • Jude Jose • Nishel Shah • \\ Isaac Opemuyi
}

Received: 13 July 2008 /Accepted: 18 July 2008/Published online: 15 August 2008

(C) Springer-Verlag 2008

\begin{abstract}
This report describes the management of a patient who developed two consecutive cornual ectopic pregnancies in a year preceded by two tubal ectopics on the same side. Interstitial pregnancy is a rare form of ectopic pregnancy with a significant mortality rate. It provides a challenge for clinicians both in their diagnosis and management. As yet, the incidence of recurrent cornual ectopic pregnancies is unknown. Currently conservative medical and surgical methods of management are favoured due to good outcomes and fewer risks. However, these methods do not protect against recurrence. In this report, we discuss the various methods of management described in literature.
\end{abstract}

Keywords Recurrent · Cornual · Pregnancy · Interstitial $\cdot$ Ectopic

\section{Case report}

A 28-year-old woman was seen in the acute gynaecology emergency unit with a 3 days history of dysuria and lower abdominal and back pain. She had amenorrhoea for 5 and $1 / 2$ weeks and had a positive pregnancy test. She was gravida 7 para $1+5$. Her first pregnancy was an ectopic

\footnotetext{
J. Jose $\cdot$ N. Shah $(\bowtie)$

Homerton Hospital,

Homerton Row,

London E9 6SR, UK

e-mail: nishelshah@hotmail.com

S. Sahoo · I. Opemuyi

Queen's Hospital,

Essex, UK
}

pregnancy which was managed in South Africa with a right partial salpingectomy. Her following three pregnancies were first trimester miscarriages and her last pregnancy was delivered at full term vaginally.

She was haemodynamically stable. On abdominal examination, there was a marked tenderness in the right iliac fossa with guarding. Vaginal examination revealed cervical excitation and right adnexal tenderness but no bleeding. Transvaginal ultrasound showed a $24-\mathrm{mm}$ mass in the right fornix, free fluid with a ground glass appearance in the pouch of Douglas and an empty uterus. An emergency laparoscopy was performed which diagnosed a ruptured ectopic pregnancy in the right tubal stump and a normal left tube and ovary (Fig. 1). A mini laparotomy and excision of the right tubal stump was carried out with good haemostasis. The patient recovered well and was discharged on the third day, post-operatively. Histology confirmed a right ruptured ectopic pregnancy.

Three months later, the same patient presented to the acute gynaecology unit with a positive pregnancy test, 5 weeks amenorrhoea and constant dull aching lower abdominal pain. She was haemodynamically stable and on examination her abdomen was soft and non-tender and there was no cervical or adnexal tenderness on vaginal examination. Transvaginal ultrasound scan showed an 18 -mm right-sided adenexal mass, free fluid in the pouch of Douglas and an empty uterus. A laparoscopy was performed, which showed that the patient was bleeding from a right cornual ectopic. The right cornual ectopic was removed laparoscopically with an endoloop and diathermy was performed for haemostasis (Fig. 2). The ectopic pregnancy sac was extruded during the procedure and sent for histology which confirmed products of conception. The post-operative BHCG was $2477 \mathrm{IU} / \mathrm{L}$. The patient was followed up over the next 2 weeks with repeat BHCGs that 


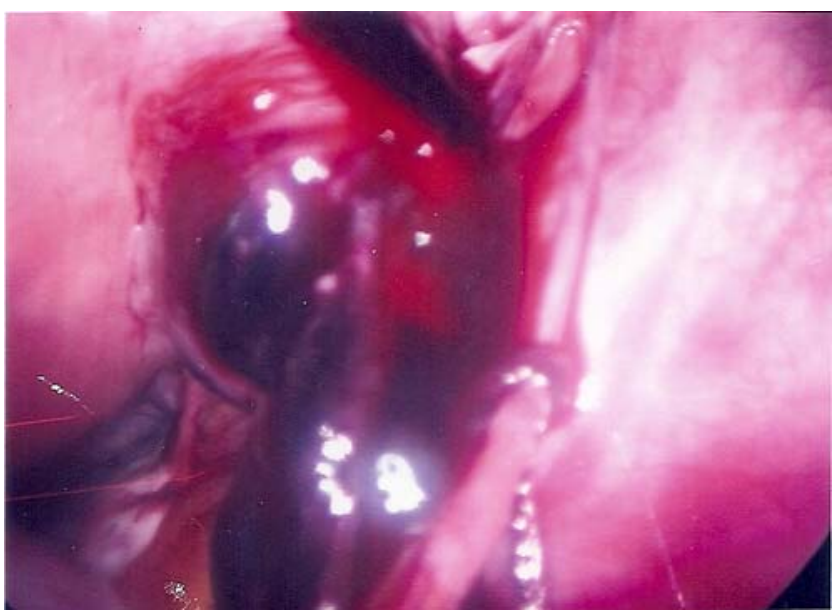

Fig. 1 Ruptured right-sided ectopic pregnancy in tubal stump

showed a rising titre. The last BHCG taken was 12,000 IU/L. At this point, a repeat transvaginal ultrasound was performed that showed a right adnexal mass with peripheral blood flow on colour Doppler suggestive of active trophoblastic tissue. Surgical management through laparotomy and excision of right cornual ectopic was offered, however, the patient declined. An alternative medical treatment was carried out, consisting of $600 \mathrm{mg}$ mifepristone given orally followed by $100 \mathrm{mg}$ of methotrexate given by intramuscular injection. The patient responded well to medical management and BHCG titres were taken every $48 \mathrm{~h}$ for 1 month. On discharge, her BHCG had fallen to $408 \mathrm{IU} / \mathrm{L}$. She restarted menstruating after 3 weeks of negative urinary pregnancy tests.

One year later, the patient presented to accident and emergency with 6 weeks of amenorrhoea. She was otherwise asymptomatic. A urinary pregnancy test was found to be positive. Owing to her previous gynaecological

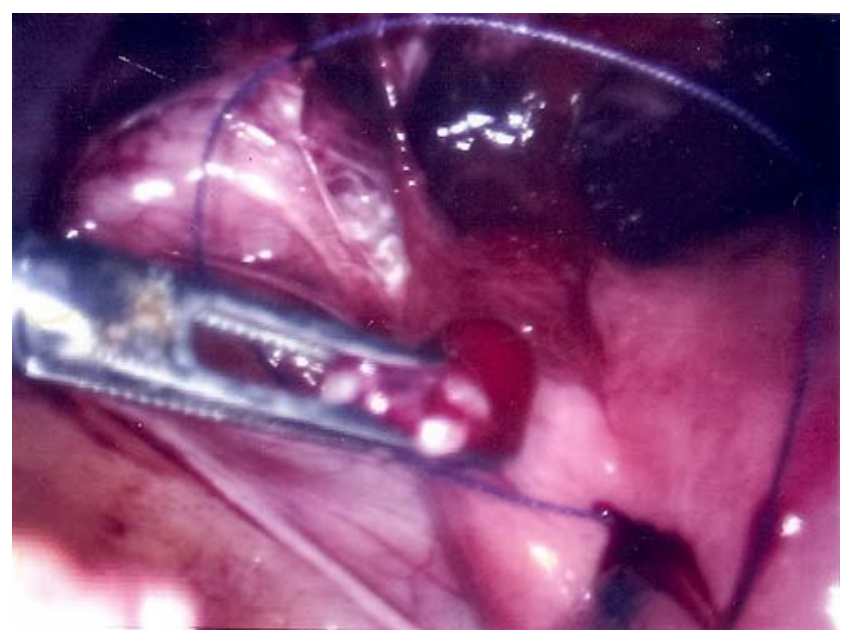

Fig. 2 Endoloop and excision of cornual ectopic history, a transvaginal ultrasound scan was performed. This showed a second consecutive right-sided cornual ectopic pregnancy. She underwent laparoscopy which confirmed the ultrasound diagnosis. Right cornual excision with an endoloop followed by diathermy for haemostasis was carried out. Hysteroscopy confirmed that the cornual pregnancy had been removed completely and showed that the cavity was not breached. Serial BHCGs were subsequently taken for the following 4 weeks that showed a decreasing trend. On discharge, the last BHCG was $152 \mathrm{IU} / \mathrm{L}$.

\section{Discussion}

Interstitial or cornual ectopic pregnancy refers to pregnancies where the conceptus implants into the proximal portion of the fallopian tube, which is within the muscular wall of the uterus [1]. Cornual ectopic pregnancies account for 1 in $2,500-5,000$ live births and $2-6 \%$ of all ectopic pregnancies [1]. They carry a significant mortality rate of $2-2.5 \%$ and account for $20 \%$ of all deaths due to ectopic pregnancies. Problems arise with cornual ectopic pregnancies because they are difficult to diagnose early and have the potential to progress into the second trimester. This is made possible due to the high-quality blood supply from branches of the uterine and ovarian vessels in addition to distensible myometrium surrounding the pregnancy. The consequences are often catastrophic with uterine rupture and profuse intraperitoneal bleeding.

Management of cornual ectopic pregnancies is another area of difficulty. Studies using medical management with methotrexate have reported failure rates approaching $35 \%$. In comparison, various surgical modalities have demonstrated better success rates (72-95\%) [2]. However, they offer no protection against recurrent cornual pregnancies and have implications for future pregnancies. As yet, the incidence of recurrent interstitial pregnancy is unknown. For tubal ectopic pregnancies, recurrence rates are $10 \%$ overall for both surgical (laparoscopic) and medical management with methotrexate [3]. In this report, we describe the management of a patient who developed two consecutive cornual ectopic pregnancies in a year preceded by two tubal ectopic pregnancies on the same side.

Diagnosis of cornual ectopic pregnancy can be made by ultrasound using the following criteria: (1) an empty uterus, (2) gestational sac seen separately and less than $1 \mathrm{~cm}$ away from the lateral-most edge of the uterine cavity, and (3) thin myometrium surrounding the sac $(<5 \mathrm{~mm})$ [4]. In addition, ultrasonographers can use the 'interstitial line sign' that has a sensitivity of $80 \%$ and a specificity of $98 \%$ [5]. On ultrasound, this is demonstrated as a thin echogenic line that extends directly up to the centre of the interstitial gestational sac [5]. 
Although the traditional method of managing cornual ectopic pregnancies has been by cornual resection, early diagnosis by ultrasound has meant greater widespread use of relatively conservative methods of surgical and medical management. A recent study by Tang et al. showed a $91 \%$ success rate in treating interstitial pregnancies using onedose intravenous methotrexate plus folinic acid with negligible side effects [6]. A randomised controlled trial by Gazvani et al. on using a combination of mifepristone and methotrexate on unruptured tubal ectopic pregnancies found faster resolution of the ectopic with the combination treatment [7]. Systemic methotrexate is not without its risks. In addition to its side effects, there remains a risk of rupture during treatment and close monitoring of serum BHCGs is required. Other forms of medical management are available including local injection of methotrexate, potassium chloride or prostaglandins under ultrasound, hysteroscopic or laparoscopic guidance $[4,8]$.

Various conservative surgical methods have been reported in the literature. Buxant et al. reported using the classical laparoscopic method of incising the cornual area after a transparietal Xylocaine-adrenaline injection, draining the contents and suturing the area [9]. However, they also used a post-operative systemic methotrexate injection [9]. Moon et al. described the vasopressin electric cauterisation method, the endoloop before evacuation of conceptus method and the encircling suture before evacuation of conceptus method [8]. However, their recommendation was that following laparoscopic management in their following pregnancies, the patients will require elective caesarean section [8]. Hysteroscopic management of cornual ectopic pregnancies has also been reported [8].

Our case report demonstrates the difficulty of managing interstitial pregnancies and avoiding recurrence. At her first presentation to us, a mini laparotomy and excision of the right tubal stump was performed. Unfortunately, the interstitial portion of a damaged tube is not removed by routine salpingectomy or, in this case, by excision of tubal stump. At her second presentation, endoloop before evacuation of conceptus method was used; however, residual trophoblasic tissue remained which was successfully treated with mifepristone and single-dose systemic methotrexate. At her third presentation, a laparoscopically performed right cornual ligation and excision was carried out with good results.

Recurrence of interstitial pregnancy is undoubtedly rare. It is important in cases of cornual ectopic pregnancies, as most conservative surgical and medical management techniques do not remove the interstitial portion of fallopian tubes, that we remain vigilant of persistent trophoblastic tissue and recurrence in future pregnancies.

\section{References}

1. Jurkovich D (2007) Ectopic pregnancy. In: Edmonds DK (ed) Dehurst's textbook of obstetrics \& gynaecology. 7th edn. Blackwell, UK

2. Canis M, Savary D, Pouly JL, Wattiez A, Mage G (2003) Ectopic pregnancy: criteria to decide between medical and surgical treatment? J Gynecol Obstet Biol Reprod (Paris) 32(7 suppl): S54-S63 (French)

3. Tay JI, Moore J, Walker JJ (2000) Regular review: ectopic pregnancy. BMJ 320:916-919

4. Timor-Tritsch IE, Monteagudo A, Matera C, Veit CR (1992) Sonographic evolution of cornual pregnancies treated without surgery. Obstet Gynecol 79:1044-1049

5. Ackerman TE, Levi CS, Dashefsky SM, Holt SC, Lindsay DJ (1993) Interstitial line sign: a sonographic finding in interstitial (cornual) ectopic pregnancy. Radiology 189:83-87

6. Tang A, Braartz D, Khoo SK (2006) A medical management of interstitial ectopic pregnancy: a 5-year clinical study. ANZJOG 46:107-111

7. Gazvani MR, Baruah DN, Alfirevic Z, Emery SJ (1998) Mifepristone in combination with methotrexate for the medical treatment of tubal pregnancy: a randomised, controlled trial. Hum Reprod 13:1987-1990

8. Pal B, Akinfenwa O, Harrington K (2003) Hysteroscopic management of cornual ectopic pregnancy. BJOG 110:879-880

9. Buxant F, Ansion MH, Noel JC, Anaf V, Simon P (2005) Laparoscopic management of a cornual ectopic pregnancy. Gynecol Surg 2:197-200 